\title{
Adequação da frequência e tipos de refeições realizadas por idosos comunitários do sul do Brasil
}

\author{
Adequacy of the frequency and types of meals consumed \\ by community-dwelling elderly folk in the south of Brazil
}

Caroline Karpinski (https://orcid.org/0000-0002-5618-7367) ${ }^{1}$

Mariana Otero Xavier (https://orcid.org/0000-0001-8791-3520) ${ }^{2}$

Ana Paula Gomes (https://orcid.org/0000-0003-2842-7340) ${ }^{3}$

Renata Moraes Bielemann (https://orcid.org/0000-0003-0202-3735) 1,2

${ }^{1}$ Faculdade de Nutrição, Universidade Federal de Pelotas (UFPel). R. Gomes Carneiro 01, Campus Anglo. 96010-610 Pelotas RS Brasil.carolinekarpinski5@ gmail.com

${ }^{2}$ Programa de Pós-

Graduação em

Epidemiologia, UFPel.

Pelotas RS Brasil.

${ }^{3}$ Faculdade de Gastronomia,

UFPel. Pelotas RS Brasil.

\begin{abstract}
The scope of this study was to describe the frequency and types of meals consumed by the elderly in Pelotas (Brazil) and factors associated with the adequacy of meal frequency. A cross-sectional study was carried out with $\geq 60$ years of age individuals. They were asked about daily meals during the week prior to the interview. The adequacy of meals was defined as recommended by the Ministry of Health (at least three main meals and two small snacks per day). The independent variables were sociodemographic, health and food routine characteristics. Prevalence ratios and 95\% confidence intervals were calculated using Poisson regression. Of the 1,438 elderly individuals interviewed, about $40 \%$ reported consuming four meals a day. Lunch was the most reported meal, followed by breakfast. A total of $30.6 \%$ of men and $38.6 \%$ of women had adequate frequency of meals ( $p=0.002$ ). Men with edentulism (total tooth loss) were 35\% more likely to eat adequately, while this probability was about $30 \%$ lower among non-white women, those without schooling and those who reported a lack of money to buy food. One in each three elderly people met the recommendation of adequate frequency of meals and some sociodemographic characteristics were negatively associated with this habit.

Key words Elderly, Eating habits, Diet, Meals
\end{abstract}

Resumo O objetivo do estudo foi descrever a frequência e tipos de refeições realizadas por idosos de Pelotas-RS e fatores associados à frequência adequada de refeições. Foi realizado estudo transversal com indivíduos $\geq 60$ anos. Questionou-se sobre as refeições diárias na semana anterior à entrevista. A adequação de refeições foi definida conforme recomendação do Ministério da Saúde (pelo menos três refeições principais e dois lanches por dia). As variáveis independentes foram as características sociodemográficas, de saúde e da rotina alimentar. Razões de prevalência e intervalos de confiança de 95\% foram calculados por regressão de Poisson. Dos 1.438 idosos entrevistados, cerca de $40 \%$ afirmaram realizar quatro refeições diárias. O almoço foi a refeição mais relatada, seguido do café da manhã. Apresentaram frequência adequada de refeições $30,6 \%$ dos homens e $38,6 \%$ das mulheres $(p=0,002)$. Homens com edentulismo (perda dentária total) tiveram 35\% maior probabilidade de adequação, enquanto essa probabilidade foi cerca de 30\% menor em mulheres de cor da pele não branca, não escolarizadas e que declararam falta de dinheiro para a compra de alimentos. Um a cada três idosos atendeu a recomendação da frequência adequada de refeições e algumas características sociodemográficas foram negativamente associadas com esse hábito.

Palavras-chave Idoso, Comportamento Alimentar, Dieta, Refeições 


\section{Introdução}

O envelhecimento da população reflete dois eventos demográficos: a queda da fecundidade, que teve seu início em meados da década de $1960^{1}$, e a queda na taxa de mortalidade, por consequência de melhora nas políticas públicas de saúde do Brasil2. Estima-se que até 2030 a população idosa dobre no país em relação ao que se identificou em 2010, atingindo em torno de 41,5 milhões de pessoas².

Com o processo de envelhecimento ocorrem mudanças na composição corporal, no funcionamento do aparelho digestivo, diminuição da função sensorial, desenvolvimento de doenças crônicas, além de efeitos secundários causados pelo uso de fármacos, os quais podem afetar o consumo alimentar dos idosos ${ }^{3,4}$. Além dos fatores biológicos, o contexto social também é determinante para a nutrição dos idosos, uma vez que o nível econômico, a cessação do trabalho, problemas psicológicos e modificações na estrutura familiar, afetam os hábitos alimentares e, consequentemente, a nutrição desse grupo etário ${ }^{3,4}$. Ademais, a capacidade funcional diminuída pode ocasionar dependência para a realização de atividades diárias relacionadas à alimentação, como a compra e a preparação dos alimentos ${ }^{5,6}$.

A diminuição na quantidade de refeições entre os idosos pode estar associada à redução fisiológica do apetite, que juntamente com a saciedade precoce, podem levar à redução na quantidade de alimentos ingeridos durante as refeições e a um maior número de lanches entre as mesmas? A saúde bucal também é relevante, pois problemas como a perda dentária podem interferir na ingestão de alimentos, bem como na escolha dos produtos a serem consumidos.

Em 2010, o Ministério da Saúde (MS) elaborou os "Dez passos para uma alimentação saudável para pessoas idosas". Um dos pontos descritos trata da distribuição dos alimentos ao longo do dia, levando em conta o contexto fisiológico desse grupo, sendo recomendada a ingestão de alimentos distribuída em cinco a seis refeições ao longo do dia, dando preferência para desjejum, almoço e jantar, com o intuito de garantir o fornecimento de nutrientes necessários, além de aporte energético adequado e bem-estar para um envelhecimento saudável ${ }^{8}$.

A ingestão alimentar em quantidades adequadas é extremamente importante para que se mantenha o funcionamento adequado do organismo, bem como para prevenir o catabolismo do tecido muscular, especialmente na população idosa que frequentemente é acometida pela redução fisiológica do apetite 9 . Ainda, o hábito de pular refeições, especialmente o café da manhã, mostrou-se associado com uma menor ingestão de micronutrientes, quando comparado à ingestão diária dessa refeição, fator de grande importância para a nutrição de grupos de risco, como os idosos $^{10}$.

Diante disso, o objetivo do presente estudo foi descrever o número de refeições e os tipos de refeições diárias realizadas por idosos comunitários moradores da zona urbana de Pelotas-RS, bem como analisar a associação entre a prevalência de adequação da frequência de refeições e variáveis sociodemográficas e de saúde.

\section{Métodos}

Trata-se de um estudo transversal de base populacional, com idosos de 60 anos ou mais, residentes da zona urbana do município de Pelotas. Os dados utilizados são provenientes do estudo "COMO VAI?" (Consórcio de Mestrado Orientado para a Valorização da Atenção ao Idoso), realizado entre janeiro e agosto de 2014.

$\mathrm{O}$ processo de amostragem foi realizado em dois estágios. Primeiramente, foi feita uma divisão dos setores censitários, de acordo com a estimativa do Instituto Brasileiro de Geografia e Estatística (IBGE), do ano de 2010, e os mesmos ordenados de acordo com a renda média dos moradores, sendo sorteados sistematicamente 133 setores. Posteriormente, houve a seleção de aproximadamente 31 domicílios por setor, de forma que fossem identificados pelo menos 12 idosos por setor. Foram excluídos da amostra, idosos institucionalizados (hospitais, instituições de longa permanência, prisões), com incapacidade física ou mental para realizar os testes solicitados ou responder os questionários sem a presença de um familiar ou cuidador. Essa incapacidade, bem como a possibilidade de fazer a entrevista com ou sem ajuda, foi avaliada pela percepção tanto da entrevistadora, quanto dos familiares/cuidadores do idoso. Também foi considerado critério de exclusão idosos que estavam em nutrição enteral.

As entrevistas foram realizadas nos domicílios dos idosos, por entrevistadoras treinadas e padronizadas, com utilização de netbooks para registro das informações. Para um controle de qualidade, foram selecionados aleatoriamente $10 \%$ dos idosos, a fim de checar a realização das entrevistas e identificar inconsistências nos dados coletados. 
As informações referentes ao número e aos tipos de refeições realizadas ao longo do dia pelos idosos foram coletadas através do relato dos entrevistados com base em respostas do tipo sim/ não para cada uma das refeições. Os idosos foram questionados se na semana anterior à entrevista habitualmente fizeram as seguintes refeições: "Na maioria dos dias, o(a) $\mathrm{Sr}(\mathrm{a})$. costuma fazer as seguintes refeições: café da manhã, lanche da manhã, almoço, lanche ou café da tarde, jantar ou café da noite, e lanche antes de dormir?". Como parâmetro para avaliar a adequação da frequência de refeições fora utilizado o primeiro passo dos "Dez passos para uma alimentação saudável para pessoas idosas", o qual recomenda a realização de pelo menos três refeições principais (café da manhã, almoço e jantar) e dois lanches saudáveis por dia8.

Como variáveis independentes, foram investigadas características socioeconômicas e demográficas (sexo, idade, cor da pele, situação conjugal, escolaridade e nível socioeconômico), fatores relacionados à alimentação, saúde bucal (edentulismo e perda dentária severa), estado nutricional e polifarmácia (uso de pelo menos cinco medicamentos diferentes concomitantemente). Quanto às características socioeconômicas e demográficas, a variável sexo foi observada pelo entrevistador e dicotomizada em masculino/feminino. A idade foi obtida em anos completos e categorizada em 60-69/70-79/ $\geq 80$. A cor da pele foi observada pelo entrevistador e posteriormente dicotomizada em branca/outras. A escolaridade, coletada em anos completos de estudo, foi categorizada em: nenhuma/1-7 anos/8 anos ou mais. A situação conjugal foi categorizada em: casado ou com companheiro/solteiro, separado ou divorciado/viúvo. Para a avaliação da situação econômica, foi utilizada como referência a classificação estabelecida pela Associação Brasileira de Empresas de Pesquisas ${ }^{11}$, a qual considera informações sobre a posse de alguns bens, além da escolaridade do chefe da família e presença de empregada doméstica, sendo posteriormente categorizada em A/B (mais ricos), C, D/E (mais pobres).

Também foram avaliadas a mudança nos hábitos alimentares devido a algum problema de saúde; a falta de dinheiro para compra de alimentos; a realização de refeições desacompanhadas; $\mathrm{e}$ a necessidade de ajuda para compra, preparação e ingestão das refeições. As quatro perguntas realizadas pertencem ao Nutrition Screening Initiative Checklist (NSI) ${ }^{12}$, instrumento de triagem que objetiva a avaliação de risco nutricional.
Com relação à perda dentária, as informações foram obtidas a partir do autorrelato, sendo verificadas pelas seguintes questões: "Quantos dentes naturais o(a) $\operatorname{Sr}(a)$. tem na parte de cima da sua boca? Por favor, se necessário, conte quantos são com auxílio da língua" e "Quantos dentes naturais o(a) $\operatorname{Sr}(a)$. tem na parte de baixo da sua boca? Por favor, se necessário, conte quantos são com auxílio da língua". Para realização da análise, foi considerada a ausência de um ou mais dentes, conforme descrição do número de dentes remanescentes, a partir do qual foram obtidas duas variáveis: edentulismo (perda dentária total) e perda dentária severa (presença de menos de nove dentes). A presença de polifarmácia (uso de cinco ou mais medicamentos) foi avaliada a partir da informação sobre o número de medicamentos em uso contínuo, prescrito por médico ou dentista, nos 15 dias anteriores à entrevista ${ }^{13}$.

A avaliação do estado nutricional, foi realizada a partir do cálculo do Índice de Massa Corporal (IMC) dos idosos [(peso (kg) / (altura (m) $)^{2}$. Para a medida de peso foi utilizada a balança eletrônica da marca Tanita, no modelo UM-080, com capacidade máxima para $150 \mathrm{~kg}$ e precisão de 100 gramas. Para a medida da altura foi utilizada a equação proposta por Chumlea ${ }^{14}$, que utiliza no cálculo a altura do joelho como estimativa da altura em pé - método mais adequado para estimar a altura nessa faixa etária, conforme recomendação da Organização Mundial da Saúde ${ }^{15}$ - a qual foi aferida utilizando-se estadiômetro infantil com medida máxima de $100 \mathrm{~cm}$ e precisão de $0,1 \mathrm{~cm}$. Foi utilizada a recomendação da Organização Mundial da Saúde ${ }^{16}$ para classificação do estado nutricional, sendo baixo peso o IMC $<18,5$ $\mathrm{kg} / \mathrm{m}^{2}$, peso adequado o IMC entre 18,5 e 24,9 $\mathrm{kg} / \mathrm{m}^{2}$, sobrepeso o IMC entre 25,0 e $29,9 \mathrm{~kg} / \mathrm{m}^{2}$, e obesidade o IMC $\geq 30,0 \mathrm{~kg} / \mathrm{m}^{2}$. Como a proporção de idosos com baixo peso foi muito baixa $(\mathrm{N}=25 ; 1,8 \%)$, estes foram analisados junto à categoria dos eutróficos.

A análise de dados foi realizada através do programa estatístico Stata 14.0 de maneira estratificada por sexo. O desfecho foi dicotomizado (sim/não) quanto à adequação da frequência de refeições diárias conforme a proposta do MS (realização das três refeições principais e pelo menos dois lanches por dia) $)^{8}$. A prevalência da adequação foi analisada utilizando-se o teste Qui-quadrado de Pearson e demonstradas as proporções com os respectivos intervalos de confiança de 95\% (IC95\%), conforme as variáveis independentes. Foi realizada regressão de Poisson para obtenção das razões de prevalências 
(RP) brutas e ajustadas e seus respectivos IC95\%. $\mathrm{Na}$ análise ajustada, seguiu-se modelo hierárquico, construído pelos autores, com três níveis. O primeiro nível (mais distal), incluiu variáveis socioeconômicas e demográficas. Por questões de colinearidade entre as variáveis "nível econômico" e "falta de dinheiro para compra de alimentos", permaneceu no modelo estatístico aquela de menor valor-p; as variáveis relacionadas aos hábitos alimentares e à saúde bucal compuseram o segundo nível, sendo que também devido à colinearidade, entre as variáveis "edentulismo" e "perda dentária severa" permaneceu no modelo final a de menor valor-p. As variáveis de estado nutricional e polifarmácia, compuseram o terceiro nível (mais proximal). As variáveis foram todas inseridas, respeitando-se o nível hierárquico, sendo mantidas na análise ajustada aquelas que apresentassem valor-p $<0,20$. $O$ ajuste estatístico foi realizado para as variáveis de mesmo nível e para as de nível superior. Foi utilizado o comando svy para considerar o efeito de delineamento amostral em todas as análises.

O projeto de estudo foi submetido e aprovado pelo Comitê de Ética em Pesquisa da Faculdade de Medicina da Universidade Federal de Pelotas (UFPel). Todos os participantes ou responsáveis assinaram Termo de Consentimento Livre e Esclarecido, sendo garantido o direito de recusa à participação no estudo e sigilo com relação aos dados informados.

\section{Resultados}

Nos 3.799 domicílios amostrados, localizou-se 1.844 idosos, sendo entrevistados 1.451 indivíduos $(78,7 \%)$ e, destes, 1.438 responderam questões inerentes ao tema do presente estudo. As perdas do estudo foram maiores em mulheres e idosos com idade entre 60 e 69 anos de ambos os sexos.

$\mathrm{Na}$ Tabela 1 pode-se observar a descrição da amostra segundo características socioeconômicas, demográficas, de saúde e relacionadas à alimentação. Do total de homens, a maior parte tinha idades entre 60 e 69 anos $(54,0 \%)$, era de cor da pele branca $(84,6 \%)$, casados/com companheiro $(76,0 \%)$, com escolaridade entre $1 \mathrm{e}$ 7 anos completos de estudo $(59,1 \%)$ e de nível socioeconômico C $(48,8 \%)$. Quanto ao estado nutricional, houve predominância de indivíduos com sobrepeso $(46,2 \%)$. Pouco menos de um terço dos homens $(30,5 \%)$ afirmou o uso contínuo de cinco ou mais medicamentos, 53,8\% possuíam perda dentária severa e $30,4 \%$ possu- íam edentulismo. Com relação à rotina alimentar, 19,1\% afirmaram já ter passado por situação de falta de dinheiro para compra de alimentos e $32,2 \%$ afirmaram mudança na alimentação devido a algum problema de saúde. A maioria afirmou não realizar as refeições desacompanhados $(78,7 \%)$ e não necessitar de ajuda para compra, preparo e realização das refeições $(87,9 \%)$.

Entre as mulheres, a maioria tinha entre 60 e 69 anos $(51,4 \%)$, era de cor da pele branca $(83,1 \%)$, com escolaridade entre 1 e 7 anos completos de estudo $(51,7 \%)$ e pertencia ao nível socioeconômico C $(54,6 \%)$. A maior parte eram viúvas $(42,6 \%)$. Mais de um terço delas apresentou sobrepeso $(39,4 \%)$ e a porcentagem de mulheres obesas $(33,2 \%)$ foi superior àquela de baixo peso/ eutrofia $(27,4 \%)$. Com relação à polifarmácia, cerca de $38,6 \%$ afirmaram o uso contínuo de cinco ou mais medicamentos. Quanto à saúde bucal, observou-se que $65,0 \%$ das mulheres possuíam perda dentária severa (incluindo edentulismo), e dentre essas, cerca de $44,3 \%$ possuíam edentulismo. O total de $20,6 \%$ da amostra feminina afirmou já ter passado pela situação de falta de dinheiro para compra de alimentos, e 29,3\% relatou mudança na alimentação devido a problema de saúde. Cerca de $34,2 \%$ das idosas afirmaram realizar as refeições desacompanhadas e $20,3 \%$ necessitar de ajuda para compra, preparo e realização das refeições (Tabela 1).

$\mathrm{Na}$ Figura 1 pode-se observar o número de refeições diárias realizadas pelos idosos. A maior parte dos homens e das mulheres afirmaram realizar o total de quatro refeições diárias $(41,2 \%$ e $38,3 \%$, respectivamente). A Figura 2 demonstra os tipos de refeições realizadas pelos idosos. O almoço foi a refeição com maior prevalência de realização, seguido do café da manhã e do jantar, em ambos os sexos. O lanche da manhã foi a única refeição que apresentou diferença entre os sexos, com maior prevalência entre as mulheres (42,5\%, IC95\%: 39,2-46,0), comparadas aos homens (32,1\%, IC95\%: 28,5-35,9). A ceia foi a refeição menos realizada pelos idosos de ambos os sexos $(24,6 \%$ dos homens e $29,6 \%$ das mulheres). Com relação à adequação da frequência de refeições diárias, 35,6\% (IC95\%: 32,7-38,6) da amostra atingiu o preconizado pelo MS, ou seja, realizavam três refeições diárias - café da manhã, almoço e jantar - e pelo menos mais dois lanches ao longo do dia, sendo 30,6\% (IC95\%: 26,9-34,5) dos homens e 38,6\% (IC95\%: 35,1-42,2) das mulheres.

Nas Tabelas 2 e 3 pode-se observar as análises bruta e ajustada, respectivamente, da associação 
Tabela 1. Descrição da amostra de idosos segundo as características socioeconômicas, demográficas, de saúde e relacionadas à alimentação $(\mathrm{n}=1.438)$. Pelotas-RS, Brasil, 2014.

\begin{tabular}{|c|c|c|c|c|}
\hline \multirow{2}{*}{ Características } & \multicolumn{2}{|r|}{ Homens } & \multicolumn{2}{|r|}{ Mulheres } \\
\hline & $\mathbf{N}$ & \% (IC95\%) & $\mathbf{N}$ & $\%(\mathrm{IC} 95 \%)$ \\
\hline \multicolumn{5}{|l|}{ Idade (anos completos) $(\mathrm{n}=1.435)$} \\
\hline $60-69$ & 288 & $54,0(49,6-58,5)$ & 464 & $51,4(48,1-54,8)$ \\
\hline $70-79$ & 170 & $31,9(28,0-36,1)$ & 286 & $31,7(28,9-34,6)$ \\
\hline$\geq 80$ & 75 & $14,1(11,3-17,3)$ & 152 & $16,9(14,5-19,5)$ \\
\hline \multicolumn{5}{|l|}{ Cor da pele $(\mathrm{n}=1.436)$} \\
\hline Branca & 451 & $84,6(80,9-87,8)$ & 750 & $83,1(79,7-85,9)$ \\
\hline Outras & 82 & $15,4(12,2-19,2)$ & 153 & $16,9(14,1-20,3)$ \\
\hline \multicolumn{5}{|l|}{ Situação conjugal ( $\mathrm{n}=1.436)$} \\
\hline Casado ou com companheiro & 405 & $76,0(72,4-79,2)$ & 354 & $39,2(35,7-42,8)$ \\
\hline Solteiro ou divorciado & 58 & $10,9(8,6-13,6)$ & 164 & $18,2(15,5-21,2)$ \\
\hline Viúvo & 70 & $13,1(10,4-16,5)$ & 385 & $42,6(39,5-45,8)$ \\
\hline \multicolumn{5}{|l|}{ Escolaridade (anos completos) $(\mathrm{n}=1.426)$} \\
\hline Nenhum & 58 & $10,9(8,2-14,5)$ & 137 & $15,3(12,7-18,3)$ \\
\hline $1-7$ anos & 313 & $59,1(53,6-64,4)$ & 463 & $51,7(47,2-56,2)$ \\
\hline 8 anos ou mais & 159 & $30,0(24,7-35,9)$ & 296 & $33,0(27,9-38,6)$ \\
\hline \multicolumn{5}{|l|}{ Nível socioeconômico $(\mathrm{ABEP})^{\mathrm{a}}(\mathrm{n}=1.363)$} \\
\hline $\mathrm{A} / \mathrm{B}$ & 199 & $39,3(33,7-45,2)$ & 280 & $32,7(27,9-37,9)$ \\
\hline $\mathrm{C}$ & 247 & $48,8(43,4-54,3)$ & 468 & $54,6(49,9-59,2)$ \\
\hline $\mathrm{D} / \mathrm{E}$ & 60 & $11,9(9,1-15,3)$ & 109 & $12,7(10,4-15,4)$ \\
\hline \multicolumn{5}{|c|}{ Falta de dinheiro para a compra de alimentos $(\mathrm{n}=1.395)$} \\
\hline Não & 427 & $83,4(78,8-87,1)$ & 701 & $79,4(76,0-82,4)$ \\
\hline $\operatorname{Sim}$ & 85 & $16,6(12,9-21,2)$ & 182 & $20,6(17,6-24,0)$ \\
\hline \multicolumn{5}{|l|}{ Edentulismo $(\mathrm{n}=1.430)$} \\
\hline Não & 369 & $69,6(65,4-73,6)$ & 501 & $55,7(52,0-59,3)$ \\
\hline Sim & 161 & $30,4(26,4-34,7)$ & 399 & $44,3(40,7-48,0)$ \\
\hline \multicolumn{5}{|c|}{ Perda dentária severa incluindo edentulismo $(\mathrm{n}=1.430)$} \\
\hline Não & 245 & $46,2(41,8-50,7)$ & 315 & $35,0(31,3-38,9)$ \\
\hline Sim & 285 & $53,8(49,3-58,2)$ & 585 & $65,0(61,1-68,7)$ \\
\hline \multicolumn{5}{|c|}{ Mudança na alimentação por problema de saúde $(\mathrm{n}=1.395)$} \\
\hline Não & 347 & $67,8(63,2-72,0)$ & 624 & $70,7(67,6-73,6)$ \\
\hline Sim & 165 & $32,2(28,0-36,8)$ & 259 & $29,3(26,4-32,4)$ \\
\hline \multicolumn{5}{|l|}{ Refeições desacompanhadas $(\mathrm{n}=1.395)$} \\
\hline Não & 403 & $78,7(75,2-81,8)$ & 581 & $65,8(62,4-69,1)$ \\
\hline Sim & 109 & $21,3(18,2-24,8)$ & 302 & $34,2(30,9-37,7)$ \\
\hline \multicolumn{5}{|c|}{ Necessidade de ajuda para comprar, cozinhar e comer $(n=1.395)$} \\
\hline Não & 450 & $87,9(84,9-90,4)$ & 704 & $79,7(77,0-82,2)$ \\
\hline Sim & 62 & $12,1(9,6-15,1)$ & 179 & $20,3(17,8-23,0)$ \\
\hline \multicolumn{5}{|l|}{ Estado nutricional $(\mathrm{OMS})^{\mathrm{b}}(\mathrm{n}=1.361)$} \\
\hline Baixo peso/eutrofia & 149 & $29,8(25,3-34,8)$ & 236 & $27,4(24,7-30,3)$ \\
\hline Sobrepeso & 231 & $46,2(41,4-51,1)$ & 339 & $39,4(36,2-42,6)$ \\
\hline Obesidade & 120 & $24,0(19,9-28,7)$ & 286 & $33,2(30,1-36,5)$ \\
\hline \multicolumn{5}{|l|}{ Polifarmácia $(\mathrm{n}=1.434)$} \\
\hline Não & 370 & $69,6(65,3-73,5)$ & 553 & $61,3(58,1-64,5)$ \\
\hline Sim & 162 & $30,5(26,5-34,7)$ & 349 & $38,7(35,5-41,9)$ \\
\hline
\end{tabular}

Fonte: Elaborado pelos autores. 


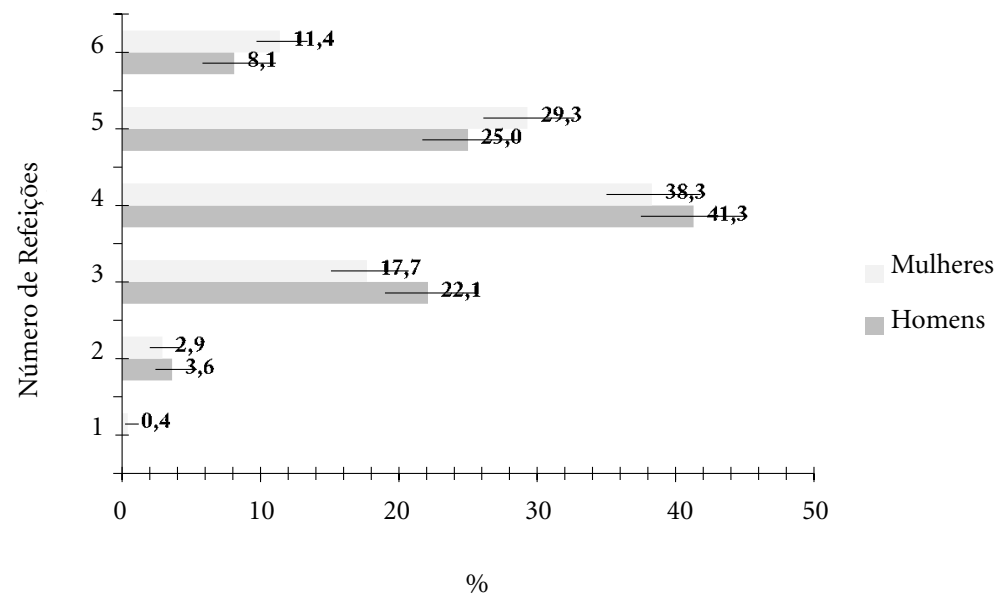

Figura 1. Número de refeições diárias realizadas por idosos não institucionalizados do Sul do Brasil. Pelotas-RS, Brasil, 2014.

Fonte: Elaborado pelos autores.

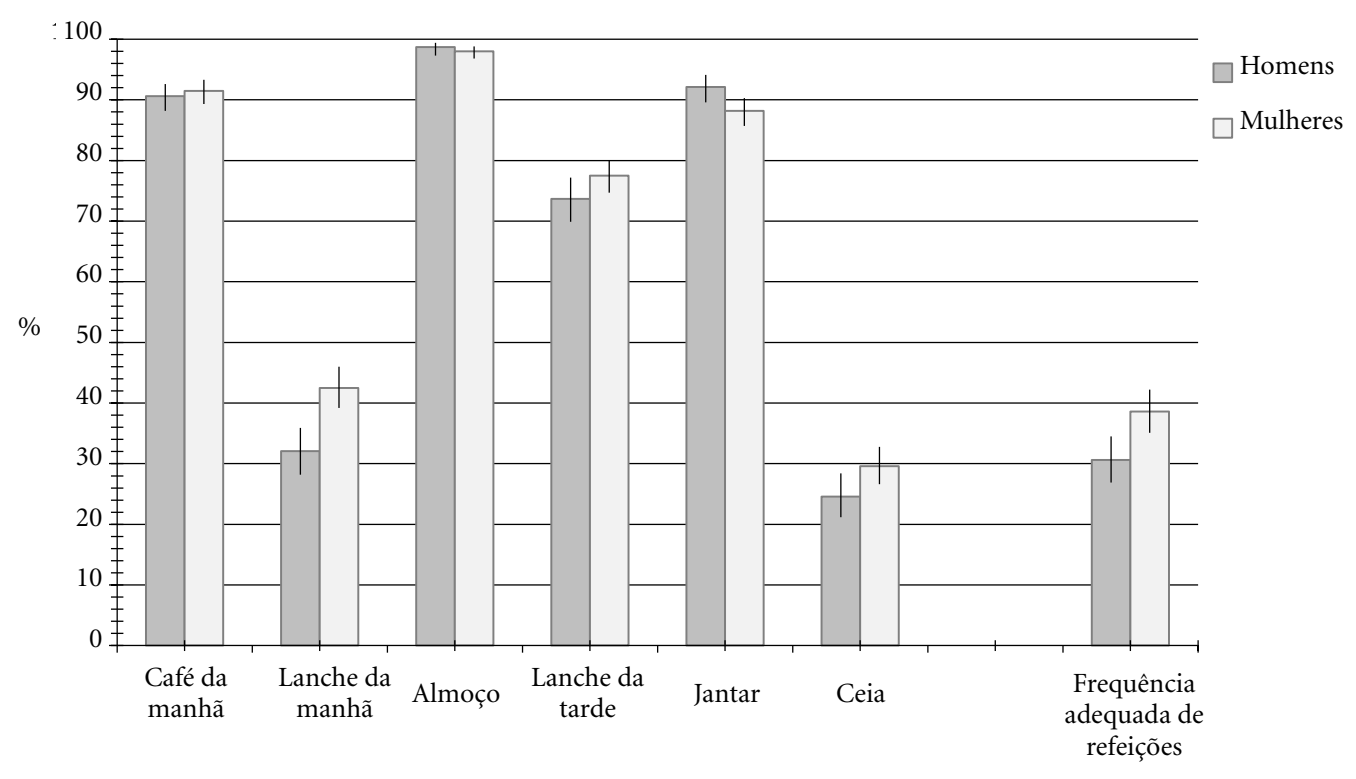

Figura 2. Tipos de refeições e adequação da frequência de refeições diárias realizadas por idosos comunitários do Sul do Brasil. Pelotas-RS, Brasil, 2014.

Fonte: Elaborado pelos autores.

de variáveis socioeconômicas, demográficas, de saúde e relacionadas à alimentação com a frequência adequada de refeições. Na análise bruta, entre os homens foi observada menor prevalência de adequação naqueles de menor nível socio- econômico (classe D/E comparada a $\mathrm{A} / \mathrm{B}$ ) e que afirmaram já ter faltado dinheiro para compra de alimentos, e maior prevalência entre aqueles que tinham edentulismo ou perda dentária severa em relação aos que não apresentavam essas 
Tabela 2. Descrição e análise bruta da associação entre idosos que realizam uma frequência adequada de refeições diárias (passo um dos dez passos do MS) e variáveis socioeconômicas, demográficas, de saúde e relacionadas a alimentação. Pelotas-RS, Brasil, 2014.

\begin{tabular}{|c|c|c|c|c|c|c|}
\hline \multirow{3}{*}{ Características } & \multicolumn{6}{|c|}{ Frequência adequada de refeições } \\
\hline & \multicolumn{3}{|c|}{ Homens } & \multicolumn{3}{|c|}{ Mulheres } \\
\hline & $\mathbf{N}(\%)$ & RP (IC95\%) & Valor-p ${ }^{\star *}$ & $\mathrm{~N}(\%)$ & RP (IC95\%) & Valor-p $^{\star *}$ \\
\hline Idade (anos completos) & & & 0,835 & & & $0,075^{\star * *}$ \\
\hline $60-69$ & $87(30,2)$ & 1,0 & & $165(35,6)$ & 1,0 & \\
\hline $70-79$ & $51(30,0)$ & $0,99(0,76-1,30)$ & & $117(40,9)$ & $1,15(0,96-1,38)$ & \\
\hline$\geq 80$ & $25(33,3)$ & $1,10(0,79-1,54)$ & & $66(43,4)$ & $1,22(0,95-1,56)$ & \\
\hline Cor da pele & & & 0,984 & & & 0,011 \\
\hline Branca & $138(30,6)$ & 1,0 & & $305(40,7)$ & 1,0 & \\
\hline Outras & $25(30,5)$ & $1,0(0,70-1,43)$ & & $43(28,1)$ & $0,69(0,52-0,92)$ & \\
\hline Situação conjugal & & & 0,643 & & & 0,514 \\
\hline $\begin{array}{l}\text { Casado ou com } \\
\text { companheiro }\end{array}$ & $126(31,1)$ & 1,0 & & $127(35,9)$ & 1,0 & \\
\hline Solteiro ou divorciado & $19(32,8)$ & $1,05(0,73-1,52)$ & & $65(39,6)$ & $1,10(0,84-1,45)$ & \\
\hline Viúvo & $18(25,7)$ & $0,83(0,54-1,27)$ & & $156(40,5)$ & $1,13(0,92-1,39)$ & \\
\hline Escolaridade (anos completos) & & & $0,322^{* * *}$ & & & 0,009 \\
\hline Nenhum & $14(24,1)$ & $0,75(0,45-1,27)$ & & $37(27,0)$ & $0,68(0,50-0,91)$ & \\
\hline $1-7$ anos & $97(31,0)$ & $0,97(0,73-1,29)$ & & $190(41,0)$ & $1,03(0,85-1,25)$ & \\
\hline 8 anos ou mais & $51(32,1)$ & 1,0 & & $118(39,9)$ & 1,0 & \\
\hline $\begin{array}{l}\text { Nível socioeconômico } \\
(\mathrm{ABEP})^{\star}\end{array}$ & & & $0,062^{* * *}$ & & & $0,026^{* * *}$ \\
\hline $\mathrm{A} / \mathrm{B}$ & $67(33,7)$ & 1,0 & & $120(42,9)$ & 1,0 & \\
\hline $\mathrm{C}$ & $76(30,8)$ & $0,91(0,67-1,24)$ & & $170(36,3)$ & $0,85(0,70-1,03)$ & \\
\hline $\mathrm{D} / \mathrm{E}$ & $11(18,3)$ & $0,54(0,31-0,97)$ & & $34(31,2)$ & $0,73(0,53-1,00)$ & \\
\hline $\begin{array}{l}\text { Falta de dinheiro para a } \\
\text { compra de alimentos }\end{array}$ & & & 0,059 & & & 0,002 \\
\hline Não & $136(31,9)$ & 1,0 & & $286(40,8)$ & 1,0 & \\
\hline Sim & $18(21,2)$ & $0,66(0,44-1,02)$ & & $48(26,4)$ & $0,65(0,50-0,84)$ & \\
\hline Edentulismo & & & 0,037 & & & 0,512 \\
\hline Não & $102(27,6)$ & 1,0 & & $198(39,5)$ & 1,0 & \\
\hline Sim & $59(36,7)$ & $1,33(1,02-1,73)$ & & $149(37,3)$ & $0,94(0,80-1,12)$ & \\
\hline
\end{tabular}

condições. Após o ajuste, os idosos de classe econômica $\mathrm{D} / \mathrm{E}$ apresentaram cerca de $50 \%$ menor prevalência de frequência adequada de refeições do que aqueles da classe $\mathrm{A} / \mathrm{B}(\mathrm{RP}=0,54$; IC95\%: $0,31-0,97)$. Ainda, idosos com edentulismo tiveram cerca de 1,3 vezes maior prevalência de frequência adequada de refeições ( $\mathrm{RP}=1,35$; IC95\%: 1,03-1,78), quando comparados aos indivíduos que não apresentavam esta condição.

Entre as mulheres, na análise bruta foram observadas menores prevalências de adequação entre aquelas de outra cor da pele que não a branca, comparadas às brancas, sem escolaridade, em relação àquelas com pelo menos 8 anos de estudo, e que declaram falta de dinheiro para compra de alimentos. Maior prevalência de 
Tabela 2. Descrição e análise bruta da associação entre idosos que realizam uma frequência adequada de refeições diárias (passo um dos dez passos do MS) e variáveis socioeconômicas, demográficas, de saúde e relacionadas a alimentação. Pelotas-RS, Brasil, 2014.

\begin{tabular}{|c|c|c|c|c|c|c|}
\hline \multirow{3}{*}{ Características } & \multicolumn{6}{|c|}{ Frequência adequada de refeições } \\
\hline & \multicolumn{3}{|c|}{ Homens } & \multicolumn{3}{|c|}{ Mulheres } \\
\hline & $\mathbf{N}(\%)$ & RP (IC95\%) & Valor-p ${ }^{\star *}$ & N (\%) & RP (IC95\%) & Valor-p ${ }^{\star *}$ \\
\hline $\begin{array}{l}\text { Perda dentária severa } \\
\text { incluindo edentulismo }\end{array}$ & & & 0,043 & & & 0,823 \\
\hline Não & $63(25,7)$ & 1,0 & & $123(39,1)$ & 1,0 & \\
\hline Sim & $98(34,4)$ & $1,34(1,01-1,77)$ & & $224(38,3)$ & $0,98(0,83-1,17)$ & \\
\hline $\begin{array}{l}\text { Mudança na alimentação } \\
\text { por problema de saúde }\end{array}$ & & & 0,372 & & & 0,131 \\
\hline Não & $100(28,8)$ & 1,0 & & $225(36,1)$ & 1,0 & \\
\hline Sim & $54(32,7)$ & $1,14(0,86-1,50)$ & & $109(42,1)$ & $1,17(0,95-1,43)$ & \\
\hline Refeições desacompanhadas & & & 0,181 & & & 0,768 \\
\hline Não & $127(31,5)$ & 1,0 & & $222(38,2)$ & 1,0 & \\
\hline Sim & $27(24,8)$ & $0,79(0,55-1,12)$ & & $112(37,1)$ & $0,97(0,79-1,19)$ & \\
\hline $\begin{array}{l}\text { Necessidade de ajuda para } \\
\text { comprar, cozinhar e comer }\end{array}$ & & & 0,473 & & & 0,473 \\
\hline Não & $133(29,6)$ & 1,0 & & $262(37,2)$ & 1,0 & \\
\hline Sim & $21(33,9)$ & $1,15(0,79-1,67)$ & & $72(40,2)$ & $1,08(0,87-1,34)$ & \\
\hline Estado nutricional & & & $0,190^{* * *}$ & & & 0,015 \\
\hline Baixo peso/eutrofia & $50(33,6)$ & 1,0 & & $91(38,6)$ & 1,0 & \\
\hline Sobrepeso & $69(29,9)$ & $0,89(0,67-1,19)$ & & $144(42,5)$ & $1,10(0,89-1,36)$ & \\
\hline Obesidade & $31(25,8)$ & $0,77(0,51-1,15)$ & & $91(31,8)$ & $0,83(0,66-1,04)$ & \\
\hline Polifarmácia & & & 0,229 & & & 0,025 \\
\hline Não & $107(28,9)$ & 1,0 & & $197(35,6)$ & 1,0 & \\
\hline Sim & $56(34,6)$ & $1,20(0,89-1,60)$ & & $151(43,3)$ & $1,21(1,02-1,44)$ & \\
\hline
\end{tabular}

*ABEP: Associação Brasileira de Empresas de Pesquisa; ${ }^{*}$ Valor-p do Teste de Wald de heterogeneidade; ${ }^{* * * V a l o r-p ~ d o ~ T e s t e ~ d e ~}$ tendência linear.

Fonte: Elaborado pelos autores.

1,01-1,50) quando comparadas àquelas que não relataram essa situação. Ainda, embora com intervalo de confiança englobando a unidade, mulheres obesas apresentaram cerca de $15 \%$ menor prevalência de adequação quanto à frequência de refeições $(\mathrm{RP}=0,85$; IC95\%: 0,68-1,06, $\mathrm{p}=0,042)$ (Tabela 3).

\section{Discussão}

Este estudo avaliou a adequação da frequência e os tipos de refeições habitualmente realizadas por idosos moradores da cidade de Pelotas-RS. Verificou-se que cerca de um terço da amostra segue a recomendação do primeiro passo dos hábitos saudáveis postulados pelo Ministério da Saúde especificamente para idosos, sendo que as mulheres apresentaram maior prevalência de adequação, comparadas aos homens. A maior parte da amostra realizava o total de quatro refeições diárias. Em ambos os sexos, o almoço foi a refeição mais realizada, seguida do café da manhã e do jantar, sendo que nove em cada dez idosos afirmaram realizar essas refeições. Entre os homens, aqueles com edentulismo apresentaram maior frequência de adequação e aqueles de menor nível econômico, menor. Nas mulheres, aquelas com outra cor da pele que não a branca, não escolarizadas e que declararam falta de dinheiro para a compra de alimentos apresentaram menor frequência adequada de refeições, enquanto que aquelas que declararam ter modificado a alimentação por problema de saúde apresentaram maior prevalência de frequência adequada de refeições diárias.

Os achados demonstraram que há um percentual expressivo de idosos que não mantêm um padrão desejável quanto à frequência de refeições diárias, para que assim fosse mantida uma nutri- 
Tabela 3. Análise ajustada da associação entre idosos que realizam uma frequência adequada de refeições diárias (passo um dos dez passos do MS) e variáveis socioeconômicas, demográficas, de saúde e relacionadas a alimentação. Pelotas-RS, Brasil, 2014.

\begin{tabular}{|c|c|c|c|c|}
\hline \multirow{3}{*}{ Características } & \multicolumn{4}{|c|}{ Frequência adequada de refeições } \\
\hline & \multicolumn{2}{|c|}{ Homens } & \multicolumn{2}{|l|}{ Mulheres } \\
\hline & RP (IC95\%) & Valor-p $^{\star *}$ & RP (IC95\%) & Valor-p $^{\star *}$ \\
\hline \multicolumn{5}{|l|}{10 Nível } \\
\hline Idade (anos completos) ${ }^{\mathrm{a}}$ & & 0,734 & & $0,069^{* * *}$ \\
\hline $60-69$ & 1,0 & & 1,0 & \\
\hline $70-79$ & $0,96(0,73-1,27)$ & & $1,18(0,98-1,44)$ & \\
\hline$\geq 80$ & $1,13(0,79-1,62)$ & & $1,22(0,94-1,58)$ & \\
\hline Cor da pele ${ }^{a}$ & & 0,969 & & 0,025 \\
\hline Branca & 1,0 & & 1,0 & \\
\hline Outras & $1,01(0,68-1,49)$ & & $0,72(0,54-0,96)$ & \\
\hline Situação conjugal ${ }^{a}$ & & 0,558 & & 0,668 \\
\hline Casado ou com companheiro & 1,0 & & 1,0 & \\
\hline Solteiro ou divorciado & $1,11(0,76-1,64)$ & & $1,13(0,87-1,30)$ & \\
\hline Viúvo & $0,82(0,53-1,27)$ & & $1,05(0,84-1,32)$ & \\
\hline Escolaridade (anos completos) ${ }^{\mathrm{a}}$ & & 0,748 & & 0,026 \\
\hline Nenhum & $0,93(0,56-1,56)$ & & $0,69(0,50-0,97)$ & \\
\hline $1-7$ anos & $1,09(0,80-1,49)$ & & $1,06(0,87-1,30)$ & \\
\hline 8 anos ou mais & 1,0 & & 1,0 & \\
\hline Nível socioeconômico $(\mathrm{ABEP})^{\star}$ & & $0,070^{* * *}$ & & $0,077^{\star * *}$ \\
\hline $\mathrm{A} / \mathrm{B}$ & 1,0 & & 1,0 & \\
\hline $\mathrm{C}$ & $0,91(0,67-1,24)$ & & $0,86(0,71-1,06)$ & \\
\hline $\mathrm{D} / \mathrm{E}$ & $0,54(0,31-0,97)$ & & $0,76(0,54-1,07)$ & \\
\hline Falta de dinheiro para a compra de alimentos ${ }^{a}$ & & 0,059 & & 0,019 \\
\hline Não & 1,0 & & 1,0 & \\
\hline $\operatorname{Sim}$ & $0,66(0,44-1,02)$ & & $0,71(0,53-0,94)$ & \\
\hline
\end{tabular}

ção adequada para um envelhecimento saudável. Quando a ingestão alimentar não é suficiente para suprir as demandas do corpo, parte da gordura corporal e massa muscular são degradadas, com o intuito de gerar energia para a manutenção das atividades normais do organismo ${ }^{9,17}$.

Embora poucos estudos tenham avaliado a realização de tipos específicos de refeições nessa faixa etária, há algumas publicações que avaliaram a frequência de realização de café da manhã entre idosos. Estudos encontraram associação positiva de realização do café da manhã com demais aspectos de saúde, como a prevenção de obesidade, melhor qualidade de vida e autonomia ${ }^{18-20}$, e maior ingestão de vitaminas e minerais ${ }^{10}$. Em um estudo realizado em Santiago (Chile) com 1.285 idosos encontrou-se que nove em cada dez idosos realizavam o café da manhã ${ }^{19}$. Já em estudo realizado no Reino Unido, quase a totalidade (99\%) dos idosos da amostra tinham o hábito de tomar café da manhã ${ }^{20}$.
Quanto a adequação da frequência de refeições que devem ser realizadas diariamente, no Brasil, Heitor et al. ${ }^{21}$, utilizando o critério de avaliação da adequação a partir dos 10 passos para alimentação saudável do idoso do Ministério da Saúde, em estudo transversal realizado com 833 idosos da zona rural de Uberaba, Minas Gerais, observaram que as mulheres, assim como no presente estudo, apresentaram maior adequação quanto à frequência de refeições ${ }^{21}$. Outro estudo, realizado por Vinholes et al. ${ }^{22}$ também em Pelotas, com adultos a partir de 20 anos de idade, também encontrou maior proporção de realização de pelo menos três refeições e um lanche por dia (antiga recomendação dos dez passos para a alimentação saudável do MS), entre as mulheres, das quais $60,8 \%$ atingiam a recomendação ${ }^{22}$.

Estudos mostram que a expectativa de vida do sexo feminino é maior do que a do sexo masculino, isso deve-se ao maior cuidado das mulheres com relação à qualidade de vida, uma vez 
Tabela 3. Análise ajustada da associação entre idosos que realizam uma frequência adequada de refeições diárias (passo um dos dez passos do MS) e variáveis socioeconômicas, demográficas, de saúde e relacionadas a alimentação. Pelotas-RS, Brasil, 2014.

\begin{tabular}{|c|c|c|c|c|}
\hline \multirow{3}{*}{ Características } & \multicolumn{4}{|c|}{ Frequência adequada de refeições } \\
\hline & \multicolumn{2}{|c|}{ Homens } & \multicolumn{2}{|c|}{ Mulheres } \\
\hline & RP (IC95\%) & Valor-p $^{\star *}$ & RP (IC95\%) & Valor-p $\mathrm{p}^{\star *}$ \\
\hline \multicolumn{5}{|l|}{ 2o Nível } \\
\hline Edentulismo $^{\mathrm{b}}$ & & 0,032 & & 0,286 \\
\hline Não & 1,0 & & 1,0 & \\
\hline Sim & $1,35(1,03-1,78)$ & & $0,90(0,74-1,09)$ & \\
\hline Perda dentária severa incluindo edentulismo ${ }^{\mathrm{b}}$ & & 0,046 & & 0,538 \\
\hline Não & 1,0 & & 1,0 & \\
\hline Sim & $1,34(1,0-1,79)$ & & $0,94(0,78-1,14)$ & \\
\hline Mudança na alimentação por problema de saúde ${ }^{b}$ & & 0,385 & & 0,042 \\
\hline Não & 1,0 & & 1,0 & \\
\hline Sim & $1,13(0,86-1,49)$ & & $1,23(1,01-1,50)$ & \\
\hline Refeições desacompanhadas ${ }^{\mathrm{b}}$ & & 0,271 & & 0,984 \\
\hline Não & 1,0 & & 1,0 & \\
\hline Sim & $0,82(0,57-1,17)$ & & $1,0(0,82-1,23)$ & \\
\hline $\begin{array}{l}\text { Necessidade de ajuda para comprar, cozinhar e } \\
\text { comer }^{\text {b }}\end{array}$ & & 0,619 & & 0,473 \\
\hline Não & 1,0 & & 1,0 & \\
\hline Sim & $1,10(0,76-1,59)$ & & $1,09(0,86-1,37)$ & \\
\hline \multicolumn{5}{|l|}{$3^{\circ}$ Nível } \\
\hline Estado nutricional $^{\mathrm{c}}$ & & $0,198^{\star * *}$ & & 0,042 \\
\hline Baixo peso/eutrofia & 1,0 & & 1,0 & \\
\hline Sobrepeso & $0,90(0,66-1,21)$ & & $1,08(0,88-1,34)$ & \\
\hline Obesidade & $0,77(0,51-1,16)$ & & $0,85(0,68-1,06)$ & \\
\hline Polifarmácia ${ }^{c}$ & & 0,524 & & 0,242 \\
\hline Não & 1,0 & & 1,0 & \\
\hline Sim & $1,11(0,81-1,52)$ & & $1,12(0,93-1,35)$ & \\
\hline
\end{tabular}

*ABEP: Associação Brasileira de Empresas de Pesquisa; ${ }^{\star *}$ Valor-p do Teste de Wald de heterogeneidade; ${ }^{\star * \star}$ Valor-p do Teste de tendência linear. ${ }^{a}$ Variáveis do $1^{\circ}$ nível (mais distal); ${ }^{b}$ Variáveis do $2^{\circ}$ nível; ${ }^{c}$ Variáveis do $3^{\circ}$ nível (mais proximal).

Fonte: Elaborado pelos autores.

que há maior procura das mesmas pelos serviços saúde, tanto para serviços de prevenção, quanto aos de assistência à saúde ${ }^{23-25}$. Da mesma forma, a preocupação com a qualidade da ingestão alimentar também é maior em mulheres de todas as idades ${ }^{26,27}$, justificando assim a maior adequação das mulheres com relação à frequência de refeições comparadas aos homens.

Como demonstrado neste estudo, fatores como a situação econômica podem estar relacionados com a frequência alimentar, especialmente entre as mulheres, embora nos homens a associação tenha seguido a mesma direção, e a significância estatística limítrofe observada pode ter ocorrido devido à menor precisão (menor proporção de homens na amostra). A insegurança alimentar é um quadro enfrentado por boa parte de população idosa brasileira e os reflexos da mesma encontram-se na saúde dessa população, que por não possuir poder aquisitivo para a compra de alimentos, muitas vezes necessita limitar não somente a qualidade, mas a frequência de refeições ${ }^{5,28}$.

Ainda no campo social, a escolaridade também é um fator determinante para o maior cuidado com a saúde. Estudos demostram que indivíduos com maior escolaridade procuram mais os serviços de saúde, além de apresentarem melhor qualidade de vida em todas as idades ${ }^{25,29}$, o que pode justificar, em parte, a relação encontrada entre menor escolaridade e menor adequação da frequência de refeições entre as mulheres idosas. Nesse contexto, destaca-se ainda a menor prevalência de adequação da frequência de refeições entre idosas de outra cor da pele que não a branca, o que provavelmente também está rela- 
cionada com a situação de vulnerabilidade social, mais frequente nesse subgrupo.

No que tange a influência da saúde bucal sobre a frequência alimentar entre idosos, embora seja reconhecido que uma saúde bucal precária pode dificultar a mastigação, assim como ocasionar a ingestão de uma dieta monótona e de baixa qualidade $\mathrm{e}^{6,28}$, no presente estudo, idosos do sexo masculino com edentulismo apresentaram maior prevalência de adequação na frequência de refeições. Estudo de revisão recente que incluiu oito estudos longitudinais para avaliar se a perda dentária afetava a ingestão alimentar e o estado nutricional de adultos concluiu que há inconsistências nos achados e, portanto, não há evidências sólidas quanto a essa relação ${ }^{30}$. No presente estudo, apenas cerca de $15 \%$ dos idosos entrevistados não faz uso de prótese - incluindo alguma dentadura, chapa, ponte ou implante (dados não apresentados), podendo ser esse um dos motivos pelos quais os resultados tenham sido controversos. Ademais, também existe a possibilidade de que aqueles com edentulismo tenham uma maior frequência de consumo de alimentos por não conseguirem ingerir um volume adequado em uma mesma refeição, o que pode ocorrer, em parte, devido à dificuldade ou demora relacionadas à mastigação.

Mesmo considerando que a dependência para atividades como a compra e o preparo de alimentos seja importante nessa faixa etária, bem como a socialização das refeições ${ }^{6}$, não foi observada associação entre a dependência para atividades relacionadas à alimentação e a realização de refeições acompanhadas com a frequência adequada de refeições. Contudo, entre mulheres que afirmaram ter modificado a alimentação devido a algum problema de saúde, a prevalência de adequação foi maior. Provavelmente esse achado está relacionado às mudanças em um conjunto de hábitos para melhorar o estilo de vida $\mathrm{e}$ a saúde em decorrência do surgimento de uma doença. Nesse sentido, destaca-se que, embora com associação limítrofe, foi observada menor prevalência de adequação da frequência de refeições entre mulheres com obesidade. É provável que estas ainda não iniciaram um processo de mudança relacionada aos hábitos alimentares e o fato de não ter uma regularidade quanto às refeições diárias pode impactar negativamente no estado nutricional ${ }^{8}$.

Embora limitações para a alimentação possam ocorrer também devido ao uso de múltiplos medicamentos, uma vez que parte da renda pode acabar sendo gasta com essa compra ${ }^{5}$, não foi en- contrada associação entre polifarmácia e frequência adequada de refeições. Todavia, a direção da associação foi positiva, indicando que aqueles que usam muitos medicamentos teriam uma maior adequação quanto à frequência de refeições diárias. Esse fato pode refletir a sobrevida com doenças crônicas ocasionada por tratamento medicamentoso adequado e também por hábitos de vida adequados, como a frequência alimentar. Cabe, porém, salientar que há indícios de que alguns medicamentos podem ter efeito direto na regulação do apetite ${ }^{31}$. Ainda, a utilização de fármacos, por outro lado, também pode ser considerada aliada a uma melhor adequação na frequência de refeições, visto que é recomendado que muitos medicamentos prescritos sejam administrados juntamente com alguma refeição, devido aos benefícios em sua absorção e para minimizar efeitos irritativos no trato gastrointestinal ${ }^{32}$.

Dentre as limitações do presente estudo, é importante citar a porcentagem de perdas e recusas (em torno de 20\%), superior no sexo feminino e naqueles com 60-69 anos. Nesse sentido, ressalta-se que podem ter influenciado a prevalência observada do desfecho subestimando o resultado encontrado com relação à frequência adequada de refeições, devido a uma melhor qualidade de saúde esperada para indivíduos mais jovens e a maior preocupação com saúde por parte das mulheres. Ainda, perguntas relacionadas à alimentação, as quais dependem da capacidade cognitiva e memória dos entrevistados, também podem ter sido prejudicadas, devido à diminuição comum dessas capacidades inerentes ao processo de envelhecimento. Por outro lado, a execução do estudo permitia a presença de um respondente chave, dependendo da condição do idoso para responder e, quanto ao questionário relacionado a ingestão alimentar, foram registradas informações referentes aos sete dias anteriores à entrevista, possibilitando a diminuição do erro de recordatório. Por último, é importante salientar que o objetivo do estudo foi restrito à análise da adequação quanto à frequência de refeições diárias recomendadas pelo MS para essa faixa etária, sem levar em conta questões como quantidade e qualidade dos alimentos consumidos em cada refeição. Assim, embora alguns idosos possam estar seguindo tal passo recomendado, não é possível afirmar que estes sejam "mais saudáveis", visto que em cada refeição pode haver consumo alimentar além ou aquém das necessidades nutricionais.

Além das estratégias acima descritas para redução de um possível efeito negativo da capacidade cognitiva dos indivíduos da amostra na 
obtenção das informações, deve ser também destacada positivamente a seleção amostral, a qual buscou abranger todas as classes econômicas presentes, visando maior representatividade na amostra. Outro ponto positivo, é a característica do estudo, que por ser de base populacional, contribui para o fornecimento de informações sobre idosos da comunidade. Além disso, por ser um grande estudo desenvolvido por pesquisadores de diversas áreas, além das características sociodemográficas, foi possível avaliar diversos domínios da vida desses idosos, os quais podem ser causas ou consequências de hábitos alimentares saudáveis.

Por fim, os resultados apontaram que os idosos consumiam, em média, quatro refeições diárias e apenas um terço deles se guia a recomendação preconizada pelo Ministério da Saúde quanto à frequência de refeições. A refeição mais prevalente foi o almoço, seguido do café da manhã e jantar. A frequência adequada de refeições foi maior entre mulheres e questões sociais como a cor da pele, a escolaridade e a condição financeira mostraram-se associadas a essa prevalência. $\mathrm{O}$ direito humano à alimentação prevê o direito de estar livre da fome e à alimentação adequada e segura, garantindo o acesso estável a uma alimentação em quantidade necessária, o que é ainda mais importante na faixa etária idosa, altamente sensível a mudanças agudas na ingestão alimentar. Futuros estudos podem trazer maiores informações acerca da qualidade das refeições dos idosos. Finalmente, ressalta-se que é fundamental que as políticas alimentares voltadas ao idoso considerem a vulnerabilidade social e a segurança alimentar dessa população.

\section{Colaboradores}

C Karpinski, MO Xavier e RM Bielemann participaram da concepção do estudo, análise, interpretação dos dados e redação do artigo. AP Gomes elaborou o instrumento de coleta de dados, supervisionou o trabalho de campo, participou da interpretação dos dados e redação do artigo. Todos as autoras revisaram criticamente o conteúdo intelectual, aprovaram a versão final a ser publicada e são responsáveis por todos os aspectos do trabalho. 


\section{Referências}

1. Simões CCS. Relações entre as ações históricas na dinâmica demográfica brasileira e os impactos decorrentes do processo de envelhecimento da população. Rio de Janeiro: IBGE; 2016

2. Instituto Brasileiro de Geografia e Estatística (IBGE). Mudança demográfica no Brasil no início do século XXI: subsídios para as projeções da população. Rio de Janeiro: IBGE; 2015.

3. Brownie $\mathrm{S}$. Why are elderly individuals at risk of nutritional deficiency? Int J Nurs Pract 2006; 12(2):110118.

4. Campos MTFS, Monteiro JBR, Ornelas APRC. Fatores que afetam o consumo alimentar e a nutrição do idoso. Rev Nutr 2000; 13(3):157-165.

5. Bernstein M. Nutritional Needs of the Older Adult. Phys Med Rehabil Clin N Am 2017; 28(4):747-766.

6. Guyonnet S, Rolland Y. Screening for Malnutrition in Older People. Clin Geriatr Med 2015; 31(3):429-437.

7. Visvanathan R. Anorexia of Aging. Clin Geriatr Med 2015; 31(3):417-427.

8. Brasil. Ministério da Saúde (MS). Secretaria de Atenção à Saúde. Alimentação saudável para a pessoa idosa: um manual para profissionais de saúde. Brasília: MS; 2009.

9. Cruz-Jentoft AJ, Kiesswetter E, Drey M, Sieber CC. Nutrition, frailty, and sarcopenia. Aging Clin Exp Res 2017; 29(1):43-48.

10. Leech RM, Worsley A, Timperio A, McNaughton SA. Understanding meal patterns: definitions, methodology and impact on nutrient intake and diet quality. Nutr Res Rev 2015; 28(1):1-21.

11. Associação Brasileira de Empresas de Pesquisa (ABEP). Alterações na aplicação do Critério Brasil, válidas a partir de 2013. São Paulo: ABEP; 2013.

12. Posner BM, Jette AM, Smith KW, Miller DR. Nutrition and health risks in the elderly: the nutrition screening initiative. Am J Public Health 1993; 83(7):972-978.

13. Nascimento R, Alvares J, Guerra AAJ, Gomes IC, Silveira MR, Costa EA, Leite SN, Costa KS, Soeiro OM, Guibu IA, Karnikowski MGO, Acurcio FA. Polypharmacy: a challenge for the primary health care of the Brazilian Unified Health System. Rev Saude Publica 2017; 51(Supl. 2):19s.

14. Chumlea WC, Guo S. Equations for predicting stature in white and black elderly individuals. J Gerontol 1992; 47(6):M197-M203.

15. World Health Organization (WHO). Physical status: the use and interpretation of anthropometry: report of a WHO Expert Committee. Genebra: WHO; 1995.

16. Organização Mundial da Saúde (OMS). Envelhecimento ativo: uma politica de saúde. Brasília: OPAS, 2005.

17. Morley JE. Pathophysiology of the anorexia of aging. Curr Opin Clin Nutr Metab Care 2013; 16(1):27-32.

18. St-Onge MP, Ard J, Baskin ML, Chiuve SE, Johnson HM, Kris-Etherton P, Varady K, American Heart Association Obesity Committee of the Council on Lifestyle and Cardiometabolic Health, Council on Cardiovascular Disease in the Young, Council on Clinical Cardiology, Stroke Council. Meal Timing and Frequency: Implications for Cardiovascular Disease Prevention: A Scientific Statement from the American Heart Association. Circulation 2017; 135(9):e96-e121.
19. García Milla P, Candia Johns P, Durán Agüero S. Asociación del consumo de desayuno y la calidad de vida en adultos mayores autonomos chilenos. Nutr Hosp 2014; 30(4):845-850.

20. Gaal S, Kerr MA, Ward M, McNulty H, Livingstone MBE. Breakfast Consumption in the UK: Patterns, Nutrient Intake and Diet Quality. A Study from the International Breakfast Research Initiative Group. Nutrients 2018; 10(8):999.

21. Heitor SFD, Rodrigues LR, Tavares DMS. Fatores associados às complicações metabólicas e alimentação em idosos da zona rural. Cien Saude Colet 2016; 21(11):3357-3366.

22. Vinholes DB, Assunção MCF, Neutzling MB. Freqüência de hábitos saudáveis de alimentação medidos a partir dos 10 Passos da Alimentação Saudável do Ministério da Saúde: Pelotas, Rio Grande do Sul, Brasil. Cad Saude Publica 2009; 25(4):791-799.

23. Abreu DM, Cesar CC, Franca EB. Gender differences in avoidable mortality in Brazil (1983-2005). Cad Saude Publica 2009; 25(12):2672-2682.

24. Borim FSA, Francisco P, Neri AL. Sociodemographic and health factors associated with mortality in community-dwelling elderly. Rev Saude Publica 2017; 51:42.

25. Pilger C, Menon MU, Mathias TAF. Utilização de serviços de saúde por idosos vivendo na comunidade. Rev Esc Enferm USP 2013; 47(1):213-220.

26. Kiefer I, Rathmanner T, Kunze M. Eating and dieting differences in men and woman. J Mens Health Gend 2005; 2(2):194-201.

27. Gomes AP, Soares AL, Goncalves H. Low diet quality in older adults: a population-based study in southern Brazil. Cien Saude Colet 2016; 21(11):3417-3428.

28. Amarya S, Singh K, Sabharwal M. Changes during aging and their association with malnutrition. J Clin Gerontol Geriatr 2015; 6(3):78-84

29. Robert SA, Cherepanov D, Palta M, Dunham NC, Feeny D, Fryback DG. Socioeconomic status and age variations in health-related quality of life: results from the national health measurement study. J Gerontol B Psychol Sci Soc Sci 2009; 64(3):378-389.

30. Gaewkhiew P, Sabbah W, Bernabe E. Does tooth loss affect dietary intake and nutritional status? A systematic review of longitudinal studies. J Dent 2017; 67:1-8.

31. Clegg ME, Williams EA. Optimizing nutrition in older people. Maturitas 2018; 112:34-38.

32. Moura MRL, Reyes FGR. Interação fármaco-nutriente: uma revisão. Rev Nutr 2002; 15:223-238.

Artigo apresentado em 19/10/2019

Aprovado em 01/06/2020

Versão final apresentada em 03/06/2020

Editores-chefes: Romeu Gomes, Antônio Augusto Moura da Silva 
